\title{
Applying Closing Phase-Angle Control Technique in Bounce Reduction of AC Permanent Magnet Contactor
}

\author{
Chieh-Tsung Chi \\ Department of Electrical Engineering, Chienkuo Technology University, No. 1, Chieh Shou N. Road, Changhua City, Taiwan \\ Correspondence should be addressed to Chieh-Tsung Chi, jih@cc.ctu.edu.tw
}

Received 9 August 2008; Revised 14 February 2009; Accepted 14 May 2009

Recommended by Ashok Goel

\begin{abstract}
A new low-cost electronic control circuit actuator is proposed for minimizing the bouncing times of an AC permanent magnet (PM) contactor after two contacts closing. The proposed new actuator overcomes the bouncing problem of an uncontrollable restrictions imposed by previously conventional AC electromagnetic (EM) contactor based on the minimization of kinetic energy prior to two contacts impact. By choosing the closing phase angle of coil voltage on purpose, the bouncing problems of the movable contact during the closing process are then overcome. The using life of contacts is then prolonged and their operating reliability is improved as well. In order to validate the feasibility and effectiveness of the proposed method here, several simulation and experimental procedures were performed on a prototype of AC PM contactor in the laboratory. Testing results actually showed that bouncing problem of contactor's contacts during the closing process was to be controlled by using the proposed technology.
\end{abstract}

Copyright (C) 2009 Chieh-Tsung Chi. This is an open access article distributed under the Creative Commons Attribution License, which permits unrestricted use, distribution, and reproduction in any medium, provided the original work is properly cited.

\section{Introduction}

Contactors have an essential place in the field of electrical devices; they are used when insulation between the control and power parts is needed. The process of the contact bounce after the movable contacts first touch the fixed contacts could be repeated several times before they reach a permanent state of contact. Contactors bouncing phenomenon during the closing process is commonly an undesirable event [1]. Especially, the lifespan and reliability of contactor depend upon the ability of their contacts to diffuse the acing heat produced during closing and opening operations $[2,3]$.

Several critical disadvantages are often to be found in conventional electromagnetic (EM) contactor, that consumes much more energy to hold armature, produces noise at lower voltage, its coil is easy to be burnt due to continual working state, and removes the abnormal dropouts results from power line disturbances like voltage sag events. In the recent years, to overcome above-mentioned disadvantages of conventional AC EM contactor [4], a new AC contactor with a permanent magnet actuator (abbreviated AC PM contactor) has been successfully developed. It has attracted more and more attention by many researchers $[5,6]$.
According to the basic collision theory [7], it is impossible that their interface can be maintained in closing status when two finite bodies under initially different velocities collide. Much work has been carried out for this undesirable phenomenon. However, all explored methods almost highlighted on minimizing the moving kinetic energy of contacts before collision or maximizing the dissipating rate of arc heat after collision [3]. To depress the making speed of contact prior to contacts impact, an intelligent AC contactors model capable of switching on at the best closing phase is presented and improved its lifespan effectively [8]. Shortly, several approaches based on intelligent algorithms were used to determine the best closing phase angle of coil voltage for different applications [9-11]. Even though many studies regarding to the contact-bounce control of conventional AC EM contactor operated in the closing process have been published, however, little attention has been paid to control the closing contact bounce related to AC PM contactor [46]. Figure 1(a) shows the standard controlling mechanism of a conventional AC EM contactor. When SW is closed, the AC EM contactor is applied to an AC voltage source and the current flows through the coil. In addition, Figure 1(b) shows the typical system configuration of a new proposed 


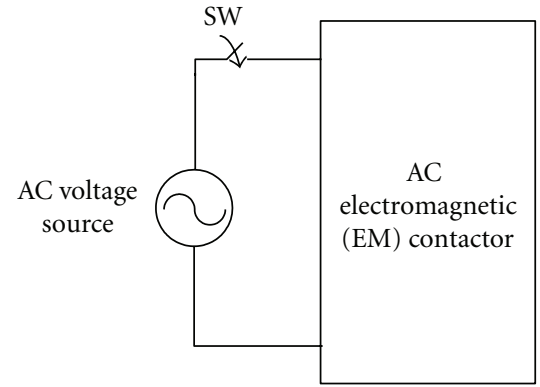

(a)

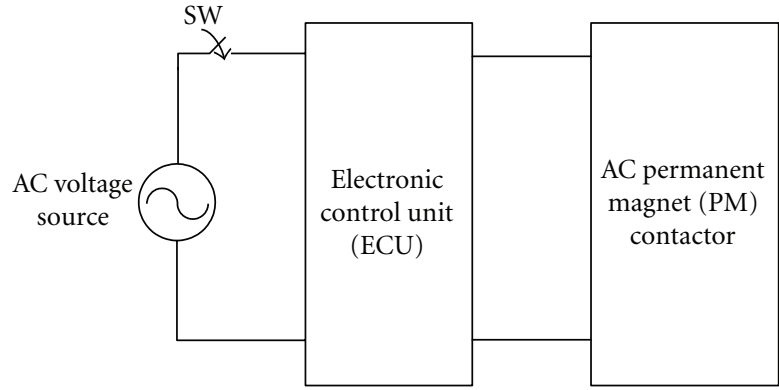

(b)

FIgURE 1: Sketch the structure of an (a) AC EM contactor; (b) AC PM contactor.

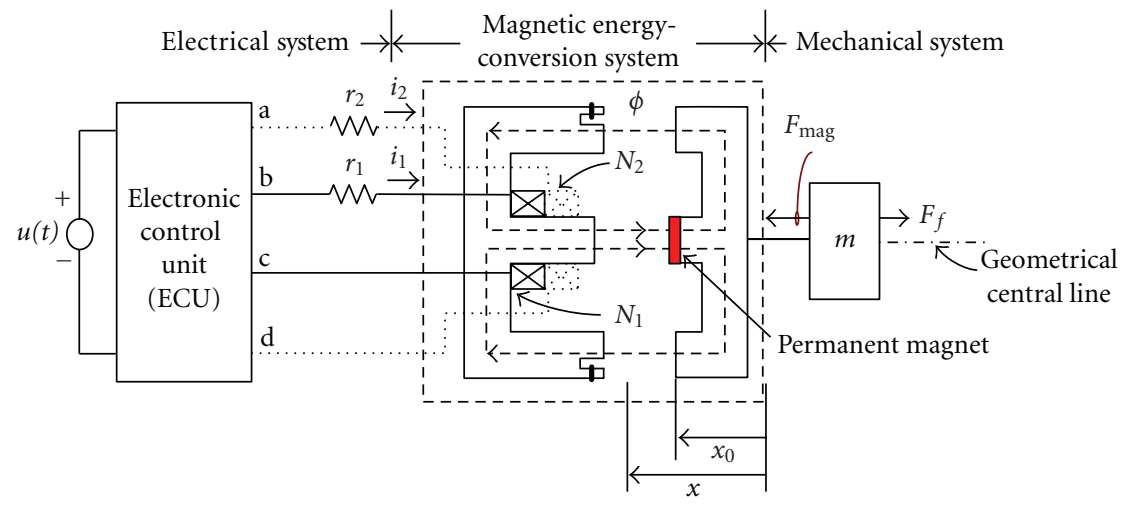

FIgURE 2: System configuration of AC PM contactor.

AC PM contactor. Note that an electronic control unit is included and connected with an $\mathrm{AC}$ voltage source and AC PM contactor coil in series. As a result of permanentmagnet force forced on armature, not only the fast transition result is achieved during the closing process, but also little energy is consumed during the holding process. Moreover, the objective of this paper is to present a controlling method for minimizing the kinetic energy of movable contacts based on purposely choosing the closing phase angle of coil voltage of AC PM contactor. Therefore, the lifespan and operating reliability are hoped to be then obtained simultaneously.

\section{Principle of Operation}

2.1. Mathematical Model. As shown in Figure 2, the structure of an AC PM contactor is composed of three subsystems: electric system, magnetic energy-conversion system, and mechanical system. The magnetic energy-conversion system includes the basic mechanism of a conventional AC EM contactor; in addition, an external permanent magnet is also arranged on armature. This permanent magnet is generally made of Nd-Fe-B material; so that its volume is small. To reduce energy loss, all iron cores in the magnetic circuit are made of the ferromagnetic material. Figure 2 depicts that the AC PM contactor is commanded by an electronic control unit (ECU). For a satisfactory ECU controlled actuator, two sets of exciting coils, such as closing coil $N_{1}$ and opening coil $N_{2}$ are together equipped with the stationary E-type iron core. The closing coil is driven during the making course, while the opening coil is driven during the breaking course.

During the closing process, the closing coil will be energized by a full-wave rectified $\mathrm{AC}$ voltage source, the equivalent circuit as shown in Figure 3(a). The resultant magnetic force imposed upon the armature within this process is the electromagnetic force combined with permanentmagnet force. Therefore, the transition time is generally shorter than conventional AC EM contactor. By employing Kirchhoff's voltage law (KVL) to the equivalent electric circuit of AC PM contactor, the voltage equation can be represented as shown below

$$
u^{*}(t)=i_{1} r_{1}+\frac{d \lambda_{1}}{d t}=\left|\sqrt{2} U_{\mathrm{rms}} \sin (\omega t)\right|,
$$

where

$u^{*}(t)$ : full-wave rectified AC sinusoidal voltage source and its frequency is twice the original applied AC sinusoidal voltage source $u(t)$;

$U_{\text {rms }}$ : root-mean-square value of the applied AC voltage source;

$r_{1}$ : resistance of the closing coil;

$i_{1}$ : current flows through the closing coil;

$\lambda_{1}$ : flux linkage produced on the closing coil, it can be represented as $\lambda_{1}=L_{1}(x) i_{1}$. 


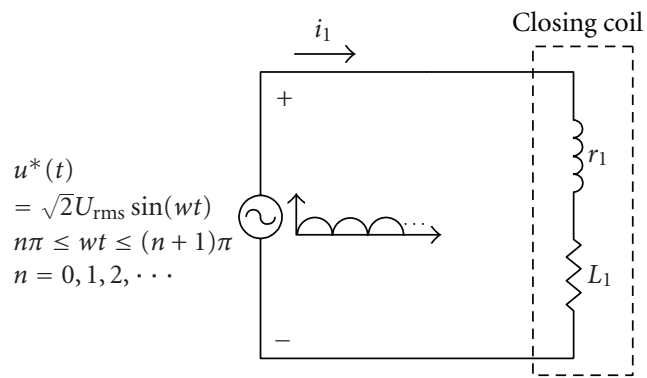

(a)

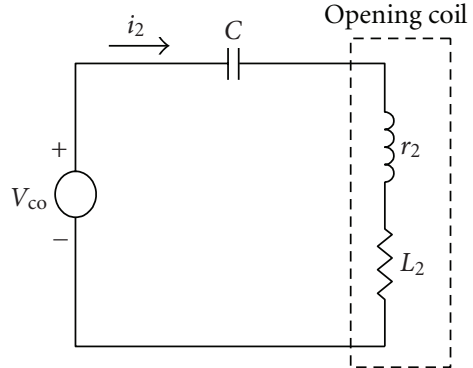

(b)

FIgURE 3: The equivalent electric circuit of AC PM contactor: (a) during the closing and holding process; (b) during the opening process.

Because the flux that flows in the magnetic circuit cannot be changed instantaneously, it must be a constant value during the closing process. Substituting the representation of linkage flux $\lambda_{1}=L_{1}(x) i_{1}$ into (1) and yields

$$
u^{*}(t)=i_{1} R^{\prime}+L_{1}(x) \frac{d i_{1}}{d t}
$$

where the generalized resistor is assumed to be defined as $R^{\prime}=r_{1}+\left(v d L_{1}\right) / d x$. As can be seen in (2), the simplified voltage equation of contactor's electric system is merely a first-order differential equation. If the initial coil current is set to be zero, that is $i_{1}(0)=0$, the complete response of coil current in (2) can be obtained and shown as follows:

$$
i_{1}(t)=-\frac{V_{m}}{|Z|} \sin (\theta-\phi) e^{-t / \tau}+\frac{V_{m}}{|Z|} \sin (w t+\theta-\phi),
$$

where $V_{m}=\sqrt{2} U_{\mathrm{rms}}$ is the amplitude of AC sinusoidal excitation. The first term in (3) is a transient part while the second term is a steady-state part. The time constant $\tau$ is $L_{1} / R^{\prime}$, the impedance $|Z|$ is $\sqrt{\left(R^{\prime}\right)^{2}+w^{2} L_{1}^{2}}$, and the power factor angle $\phi$ is $\tan ^{-1}\left(w L_{1} / R^{\prime}\right)$, respectively. It is evident that the coil current $i_{1}(t)$ includes a dc offset when the circuit is being energized at a point of the sinusoidal wave other than at $\theta=\phi$, and this dc-offset component decays exponentially at a rate equal to $L_{1} / R^{\prime}$ time constant of the electric portion of AC PM contactor. In addition, as shown in Figure 3(b), a negative electromagnetic force will be produced to overcome the holding force of the permanent magnet when the opening coil is energized by a breaking voltage across the capacitor $\mathrm{C}$.

2.2. Equivalent Magnetic Circuit Analysis. The geometry and equivalent magnetic circuit of the developed AC PM contactor is shown in Figure 4. Clearly, the considered magnetic mechanism of the AC PM contactor is symmetrical. Therefore, the magnetic circuit analysis can be then easily simplified. Compared with the conventional AC EM contactor, an additional permanent magnet should be arranged on the fixed E-type core. By applying the magnetic circuit analysis technique, the magnetic equations of AC PM contactor can be written below

$$
\begin{gathered}
\left(\Re_{1}+\mathfrak{R}_{x 1}+\Re_{3}+\Re_{x 3}+\Re_{4}\right) \phi_{3}-\left(\Re_{3}+\Re_{x 3}\right) \phi_{2}=N_{1} i_{1}+F_{\mathrm{mag}} \\
-\left(\mathfrak{R}_{3}+\mathfrak{R}_{x 3}\right) \phi_{3}+\left(\Re_{2}+\mathfrak{R}_{x 2}+\mathfrak{R}_{3}+\mathfrak{R}_{x 3}\right) \phi_{2}=i_{2},
\end{gathered}
$$

where the reluctances in each part of magnetic circuit are first calculated respectively by using reluctance principle. They are expressed as follows:

$$
\begin{gathered}
\mathfrak{R}_{1}=\frac{l_{1}+l_{1}^{\prime}+l_{3}}{u_{0} u_{r} A_{1}}, \quad \Re_{2}=\frac{l_{2}}{2 u_{0} u_{r} A_{2}}, \quad \Re_{3}=\frac{l_{2}}{2 u_{0} u_{r} A_{3}} \\
\mathfrak{R}_{x 1}=\frac{x+e}{u_{0} A_{1}}, \Re_{x 2}=\frac{x}{2 u_{0} A_{2}}, \mathfrak{R}_{x 3}=\frac{x}{2 u_{0} A_{3}}, \mathfrak{R}_{4}=\frac{l_{3}^{\prime}}{u_{0} u_{r} A_{1}} .
\end{gathered}
$$

The reluctance in each part of magnetic circuit is generally a function of the average length of individual magnetic circuit. Analog to the total resistance in the electrical circuit, the total reluctance $R(x)$ in the magnetic circuit can be obtained by using the similar calculating procedures. Shortly, the equivalent inductance value $L(x)$ across the coil can also be derived by the following formulas

$$
L(x)=\frac{N^{2}}{R(x)},
$$

where $N$ is the number of windings of coil. The relationship among flux $\phi$, flux density $\vec{B}$ and cross-sectional area of iron core is given by

$$
\phi=\int \vec{B} \cdot d \vec{S}=\vec{B} \cdot \vec{S}
$$

where $\vec{B}$ and $\vec{S}$ are the vector quantities whose magnitude is $B$ and $S$, respectively.

2.3. Determination of Electromagnetic Force. The electromagnetic force $F_{e}$ (not magnetic force $F_{\mathrm{mag}}$ ) is a function of coil current and armature displacement. If the armature displacement is held at a constant, then the variation of mechanical energy is equivalent to zero. The stored magnetic 


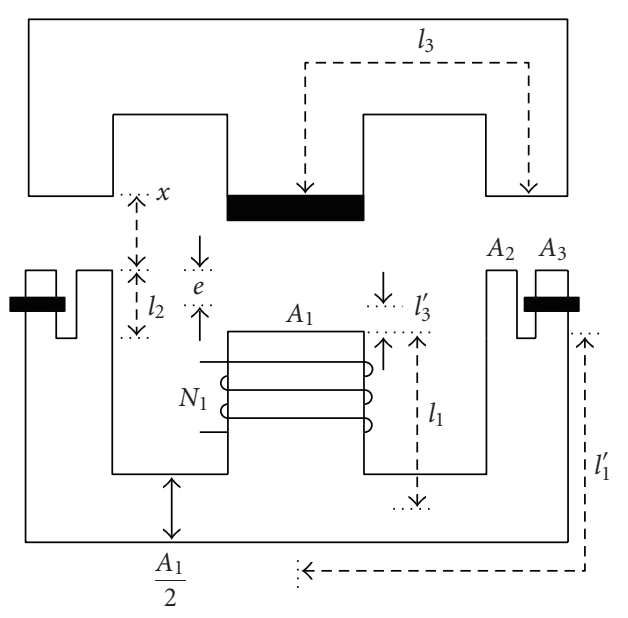

(a)

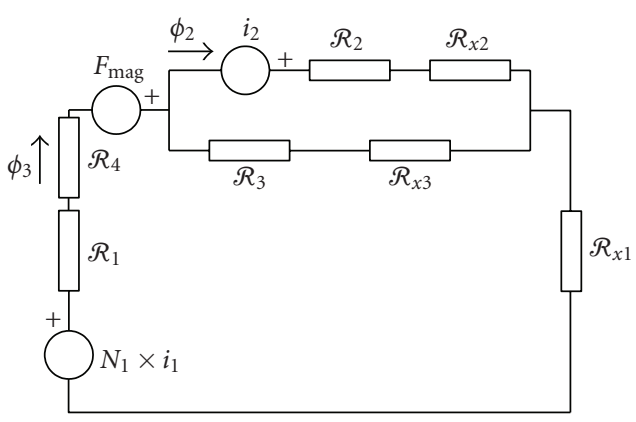

(b)

FIgURE 4: Developed AC PM contactor, (a) sketch of the geometry and (b) equivalent magnetic circuit.

coenergy in the magnetic energy-conversion system can be expressed as [12]

$$
\begin{aligned}
W_{c}^{\prime}(\lambda, i) & =\int_{0}^{\lambda} \frac{\zeta}{L(x)} d \zeta \\
& =\frac{\lambda^{2}}{2 L(x)},
\end{aligned}
$$

where $\zeta$ is the dummy variable of integration. Since the relationship between coil current and flux linkage is given by $i(\lambda, x)=\lambda / L(x)$, the electromagnetic force is the derivative of the stored magnetic coenergy. The electromagnetic force is the derivative of the stored magnetic coenergy. Thus,

$$
\begin{aligned}
F_{e} & =\frac{\partial W_{c}^{\prime}(\lambda, i)}{\partial x} \\
& =\frac{\partial}{\partial x}\left[\int_{v}\left(\int_{0}^{H} \vec{B} \cdot d \vec{H}\right) d v\right] \\
& =\frac{1}{2} i^{2} \frac{\partial L(x)}{\partial x}
\end{aligned}
$$

According to [6], in fact, no matter how the permanent magnet is arranged on armature or the fixed iron core of magnetic circuit, the effect of the permanent-magnet force upon armature is near to equal. The resultant force imposed upon armature includes gravitational force, friction force, and magnetic force. Figure 2 shows that the normal line of installation platform is commonly parallel with the geometrical central line of contactor mechanism and leads to the gravitational force component that almost does not influence on the resultant force. Therefore, the final resultant force can be simplified and expressed as shown below

$$
F=F_{\operatorname{mag}}-F_{f}
$$

where $F_{\text {mag }}$ is the magnetic force which consists of electromagnetic force and permanent-magnet force during the closing and opening processes, however, it merely includes the holding force of permanent magnet during holding process. $F_{f}$ only represents the spring antiforce. The equation governing the motion of armature can be directly formulated from Newton's law of motion as shown below

$$
F_{\mathrm{mag}}-F_{f}=m \frac{d^{2} x}{d t^{2}}
$$

where $m$ is the mass of armature. Based on work-energy theorem in physics [7], the resultant mechanical work done by armature can be presented as follows:

$$
\begin{aligned}
W_{m} & =-\int_{x}^{x_{0}} F_{\mathrm{mag}} d x \\
& =\int_{v_{0}}^{v} m v d v \\
& =\frac{1}{2} m v^{2}-\frac{1}{2} m v_{0}^{2}=\Delta E_{k},
\end{aligned}
$$

where the initial velocity of armature $v_{0}$ is zero since the armature is stationary at opening position, the final kinetic energy of armature or movable contacts $E_{k}=\left(m v^{2}\right) / 2$ is completely determined by the final velocity of armature $v$ before two contacts impact.

\section{Theoretical Analysis of Closing Contact Bounce}

By Newton's third law, the changing rate of a system momentum varies proportionally with the resultant force imposed upon the system and is in the direction of that force. It follows that the vector change in momentum of either system, in any time interval, is equal in magnitude and opposite in direction to the vector change in momentum of the other. The net change in momentum of the system is therefore zero. That is,

$$
\sum P_{f}=\sum P_{i}
$$




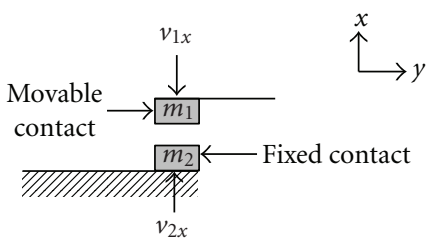

(a)

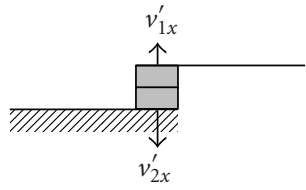

(b)
FIgURE 5: The sketch of movable contact and fixed contact (a) before collision (b) after collision.

where $P_{i}$ and $P_{f}$ are stand for the linear momentum of movable contact before and after two contacts impact, respectively. Since the linear momentum of movable contact is conservative, it is to be held a constant value before and after two contacts impact. Currently, the contact system of contactor can be viewed as consisting of two parts, a movable contact and fixed contact. The mass of movable contact is $m_{1}$ and its initial moving velocity is $v_{1 x}$. In contrast, the mass of fixed contact is $m_{2}$ and its initial velocity $v_{2 x}$ is zero, that is, stationary. If the movable contact is collided with the fixed contact along with a straight line, the final velocities of these contacts can be written as follows:

$$
m_{1} v_{1 x}^{\prime}+m_{2} v_{2 x}^{\prime}=m_{1} v_{1 x}
$$

Figure 5 shows the sketch of the movable contact and fixed contact before and after two contacts impact. The total kinetic energy of contact system should be maintained at a constant value due to the conservative principle. In other words, the initial total kinetic energy value of contact system is equivalent to the one of final total kinetic energy value. Therefore, it can be represented by

$$
\frac{1}{2} m_{1}\left(v_{1 x}^{\prime}\right)^{2}+\frac{1}{2} m_{2}\left(v_{2 x}^{\prime}\right)^{2}=\frac{1}{2} m_{1} v_{1 x}^{2} .
$$

From the expressions of linear momentum and kinetic energy indicated in (14) and (15), respectively, we can calculate the final velocities of both movable contact and fixed contact after two contacts impact be which can given as follows:

$$
\begin{aligned}
& v_{2 x}^{\prime}=\frac{2 m_{1}}{m_{1}+m_{2}} v_{1 x} \\
& v_{1 x}^{\prime}=\frac{m_{1}-m_{2}}{m_{1}+m_{2}} v_{1 x} .
\end{aligned}
$$

The fixed contact is always arranged on the fixed mechanical frame of the contactor and as a result assuming $m_{1} \ll m_{2}$ is reasonable. Because the fixed contact is always stationary after two contacts impact, the final velocity of fixed contact depicted in (17) equals zero. In contrast, the final velocity of movable contact is equal in magnitude and opposite in direction of initial velocity. This means that the linear momentum produced by movable contact will act on the contact spring system with a linear momentum $m_{1} v_{1 x}^{\prime}$. Thus, the stretched and compressed actions of contact spring can be repeated for many times before attaining permanent state, that is, contact bounce. As we known, the contact bounce mainly depends on three influencing factors, such as the characteristic of contact spring which is not allowed to change for a existing products, the current flows through the contact which is also not controllable as long as it is lower than rating value, and controlling the moving velocity of movable contact prior to the impact. Obviously, the last method is more feasible than other approaches, and it is easy to be achieved by using controlling approach.

\section{Electronic Control Unit}

4.1. Control Strategy. As we know, if an AC PM contactor is operated in the closing process, the armature is not only forced by an uncontrollable permanent-magnet force, but also forced by a controllable electromagnetic force. Therefore, the desired dynamic resultant magnetic force value imposed upon armature will be derived by controlling the one of independent variable of contactor, such as coil current. However, the coil current is a function of the closing phase angle of applied AC sinusoidal voltage source. In other words, if we carefully select a proper closing phase angle of AC sinusoidal voltage source, an anticipated value of coil current will be obtained and leads to the desired value of resultant magnetic force. This result can also be obtained from those representations shown in (3), (9), and (11).

4.2. Implementation of Control Circuit. Figure 6 shows a complete electronic control circuit for the control of proposed AC PM contactor, it is referred to as an electronic control unit (ECU) here. The operation process of ECU is determined by the value of applied coil voltage. The ECU is composed of several simple digital and analog components. Therefore, the manufacturing cost of ECU is inexpensive. The ECU is also designed for controlling the closing contact bounce, improving robustness, and monitoring other dynamic behaviors of AC PM contactor. The remainder of this section will be used to describe the operation of the ECU gradually. Each functional block shown in Figure 6 will be described in a separate paragraph.

(1) Rectifier Circuit. The input portion of the ECU is equipped with a full-wave bridge rectifier operating from an AC sinusoidal voltage source $u(t)$ as indicated in Figure 6. It is responsible for converting the AC sinusoidal voltage source to a dc pulsating voltage $u^{*}(t)$, as denoted in Figure 3. The rectified AC voltage source $u^{*}(t)$ has the same amplitude as $u(t)$. However, the frequency of $u^{*}(t)$ is two times $u(t)$. A metal-oxide varistor (MOV) (it is included in ECU, but it is not drawn in Figure 6) is connected with the input of full-wave bridge rectifier in parallel to prevent a suddenly produced higher transient over-voltage in applied AC voltage source from damaging the ECU.

(2) Coil Voltage and Current Detectors. Part G in Figure 6 depicts that the designed coil voltage detector includes two resistors, R19 and R21. It is connected with the full-wave rectified AC voltage source in parallel. First, the sampled coil 


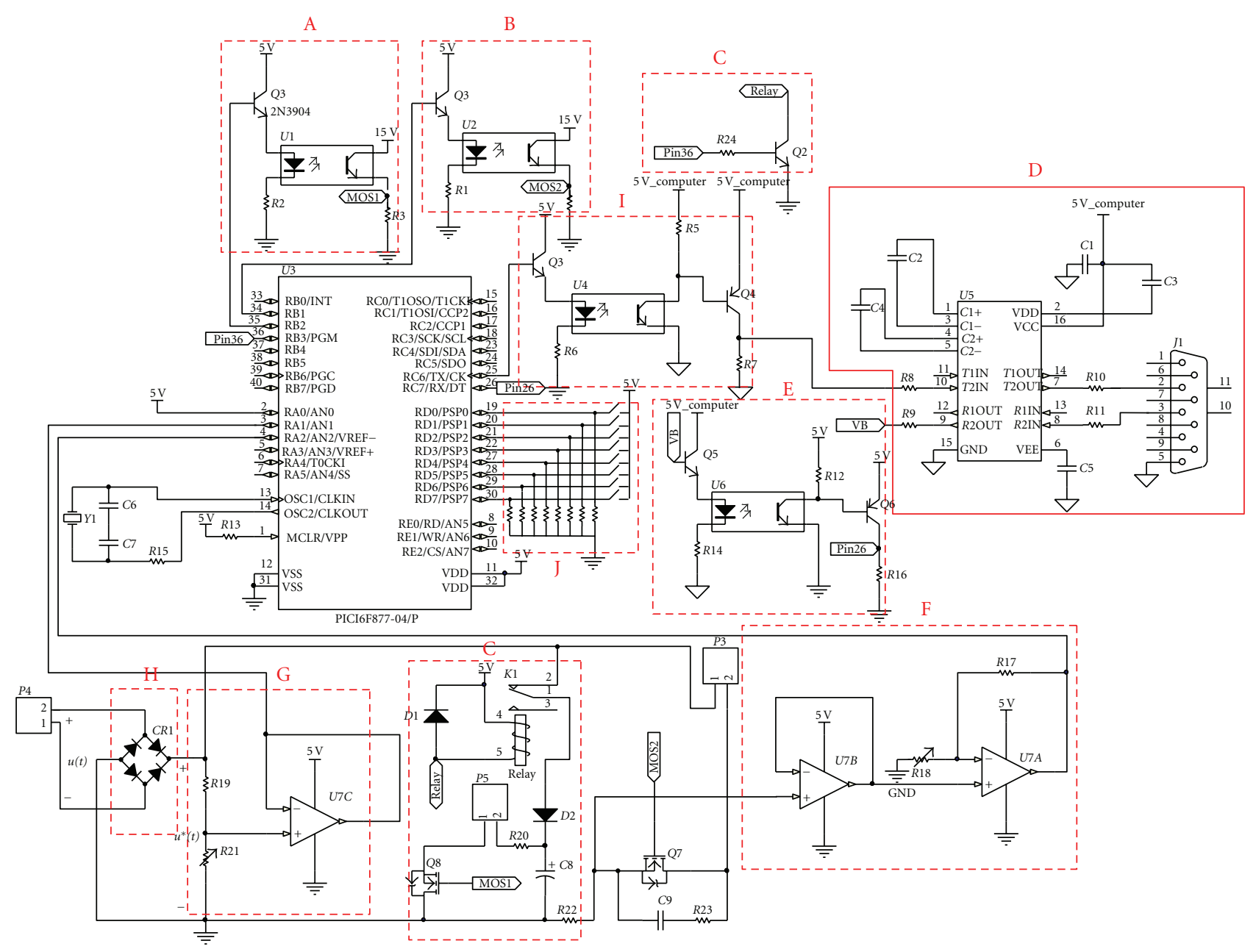

Figure 6: It shows the schematic diagram of a microcontroller-based ECU.

voltage is amplified by a unity amplifier to increase the load impedance, and then is sampled and fed to microcontroller. In addition, a coil current detector, which consists of a resistor R22, is connected with the coil in series. The resistance of R22 should be as small as possible to avoid affecting the coil current value; hence, it is designed to only have $20 \mathrm{~m} \Omega$. The voltage across R 22 is proportional to the coil current. Therefore, the voltage across R22 is sampled and justified by microcontroller through two amplifying stages, as the part F shown in Figure 6, the coil current value is then easy to be calculated by using Ohm's law.

(3) Making Signal Generator and Driver. After the voltage value of the dc supply is stable, the coil voltage begins to be read and justified by microcontroller. If the sampled coil voltage attains the desired minimum closing voltage value of AC PM contactor, microcontroller begins producing a making signal according to the setting of closing phase angle of AC voltage source shown in part J of Figure 6, which is a logical high signal over a period of closing time, to close the contactor. In general, the logical making signal should be first amplified by a voltage amplifier, as shown in the part B in
Figure 6, and then used to conduct the power MOSFET Q7. After the closing process has been completed, this making signal will disappear immediately.

(4) Breaking Signal Generator and Driver. Normally, the electrolytic capacitor $\mathrm{C} 8$ should be charged to the amplitude of $\mathrm{AC}$ voltage source before opening process starts, that is, $\sqrt{2} U_{\text {rms }}$, this is also called a breaking voltage. If the coil voltage value is lower than the maximum releasing voltage of the AC PM contactor, microcontroller begins to generate a logical breaking signal and amplified to drive power MOSFET Q8 on for performing the opening process.

(5) Serial-Port Interface. To provide field and remote controlling capability with the ECU, there is a serial-port communication interface also included in the ECU, as shown in part D in Figure 6. Integrated circuit U5 is combined with its interface circuit to form a signal converter from RS-232 level to logical type or vice versa. Moreover, a photo-coupler is installed between microcontroller and series port interface for increasing the operating safety of ECU. 


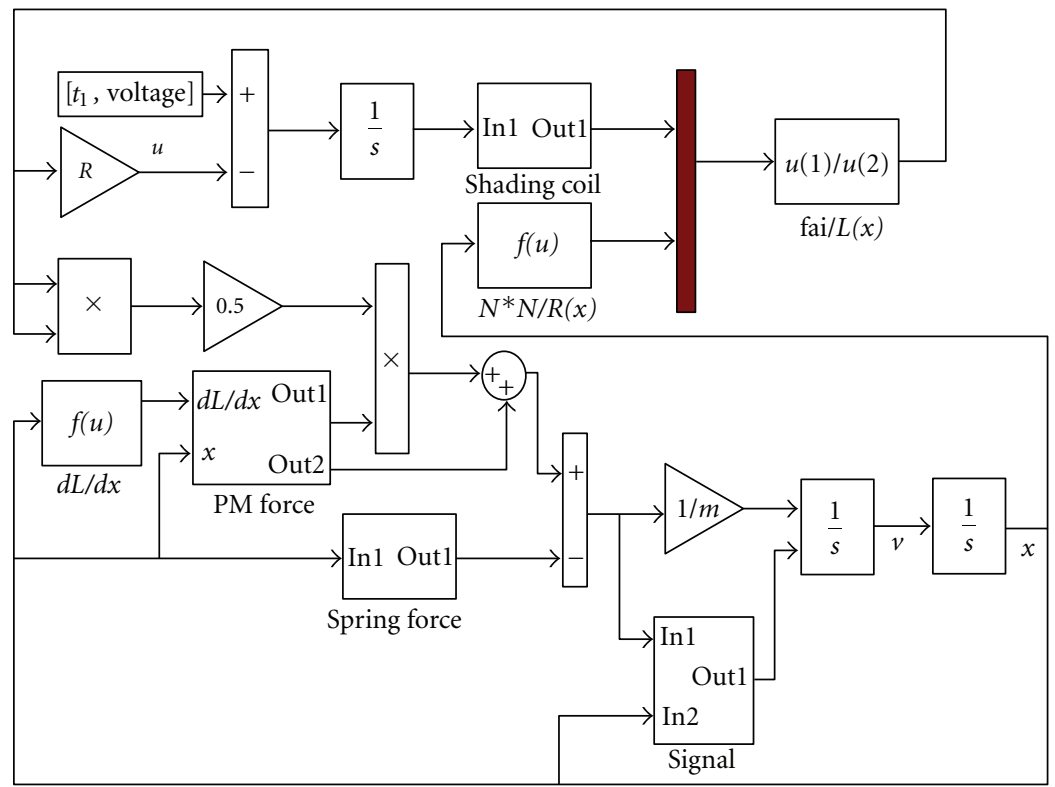

Figure 7: It shows the complete simulation model.

\section{Simulation and Experimental Results and Discussions}

For convenient conducting of the relevant experiments, an experimental contactor prototype has been established in our laboratory. This contactor prototype is allowed to be supplied to a rated rms voltage $220 \mathrm{~V}$ of AC voltage source. The capacity contactor contact is $5.5 \mathrm{KW}$ and its nominal value of the coil current is $24 \mathrm{~A}$. The number of windings is 3750 turns, the resistance across two coils' terminals is $285 \Omega$, and the mass of armature is $0.115 \mathrm{Kg}$. The contact's gap is $4 \mathrm{~mm}$, while the air gap between the armature and fixed iron core is about $6 \mathrm{~mm}$. A permanent magnet is arranged on the central leg of armature.

5.1. Model Setup and Verification of Feasibility. The dynamic simulation model of the AC PM contactor, which was implemented by Matlab/Simulink software, had been established by using the governing equivalent electrical and mechanical equations. Figure 7 shows the completed simulation model of AC PM model.

In theory, all the dynamic behavior of AC PM contactor could be characterized directly by two independent variables, such as the displacement of armature and coil current. Figures 8(a) and 8(b) show all time-varying waveforms of the armature displacement and coil current during the closing, holding and opening processes obtained by simulation model while was matched with those ones by contactor prototype well. Observing the simulated coil-current curve during the closing process, as shown in Figure 8(b), a larger coil-current difference was produced between the measured coil current and the simulated coil current from $t=0.04$ seconds to $t=0.055$ seconds. This was mainly caused by some small mechanical friction forces of contactor to be ignored. When the operating state of AC PM contactor had entered into holding process, we could see that the coil current was near to zero as the applied AC voltage source was removed. Only a little input electrical energy will be absorbed by the ECU. This is the reason why the AC PM contactor has an outstanding energy-saving performance than the other electromagnetic contactors. Figures $8(\mathrm{e})$ and $8(\mathrm{f})$ showed the displacement of armature and coil current of AC PM contactor during the opening process. The coil current first produced by a maximum negative peak value was used to generate a sufficient electromagnetic force for overcoming the holding force produced by permanent magnet. Shortly, the current flowing through the opening coil was then attenuated exponentially to zero because the disengagement of contacts had been completed. To reduce the opening arcs produced between movable contacts and fixed contacts, the total opening time duration of AC PM contactor should be controlled as little as possible.

\subsection{Implementation of Moving Velocity Control of Armature.} As mentioned above, in addition to the fact that basic mechanism of AC PM contactor must be equipped with a permanent magnet on armature, an ECU is also needed and used to control the operation of contactor under different operating processes. Figures 9(a) and 9(b) show the typical timing sequences of AC PM contactor that is operated in the closing process and opening processes, respectively. Figure 9(c) demonstrates an incorporated control flow chart of microcontroller. Note that these functions of microcontroller have already been built in ECU. Figure 9(a) depicts that microcontroller should first sample the zero crossing point of AC applied voltage source. A closing phase angle of the AC PM contactor was selected. Namely, AC PM contactor was assumed to be triggered by pin $\mathrm{RB} 1$ of microcontroller at point $\mathrm{B}$ and operated in the closing process. The closing period of the AC PM contactor was maintained by $t_{2}$ time. 


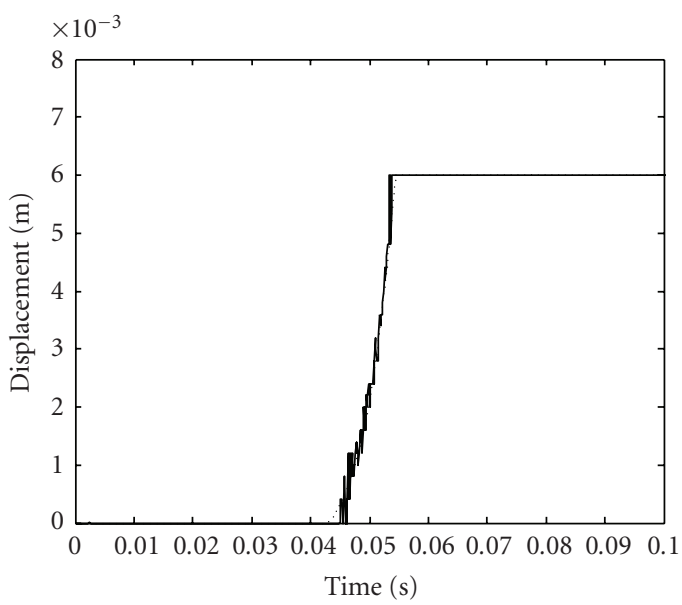

(a)

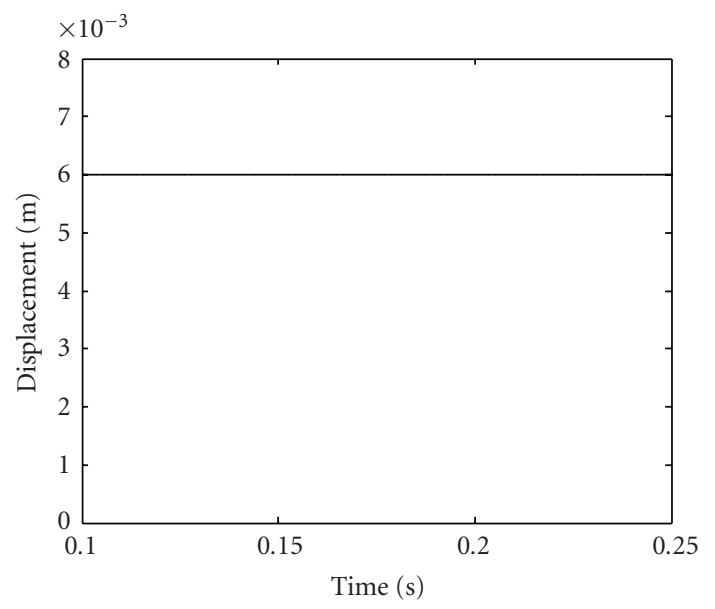

(c)

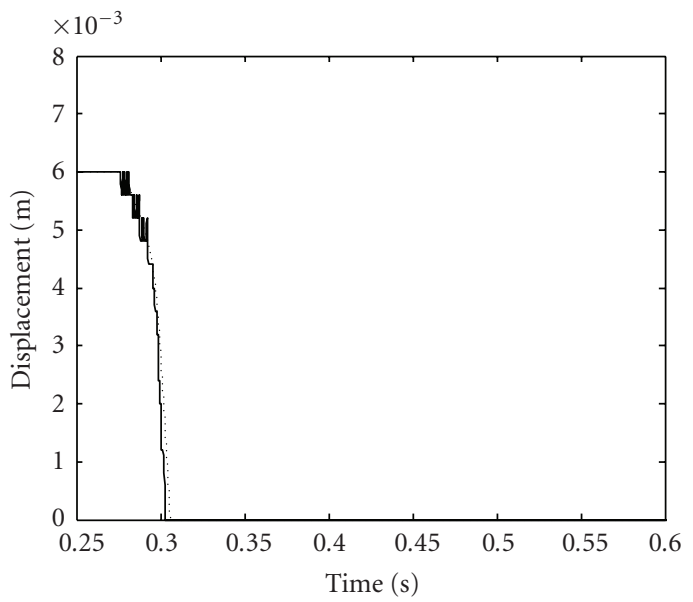

- Experiment Simulation

(e)

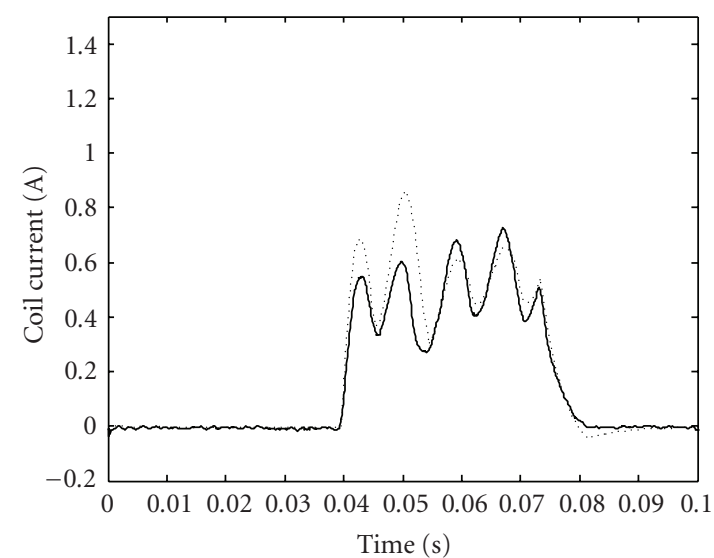

(b)

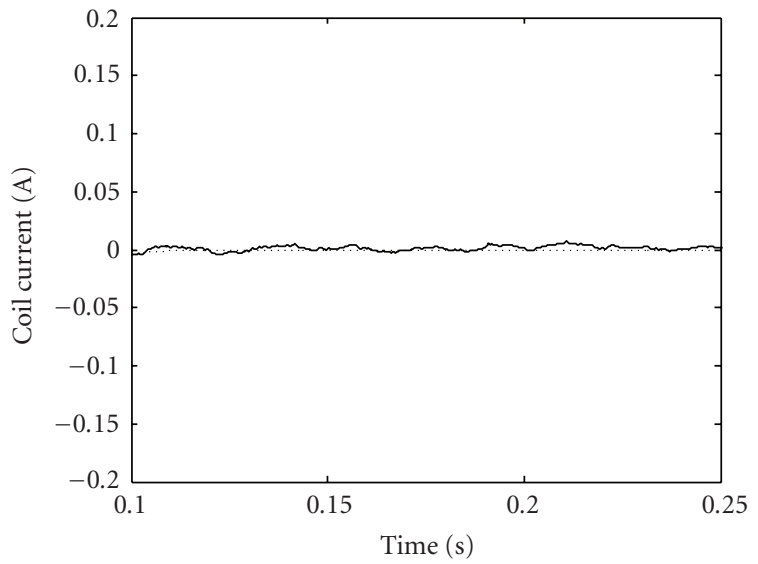

(d)

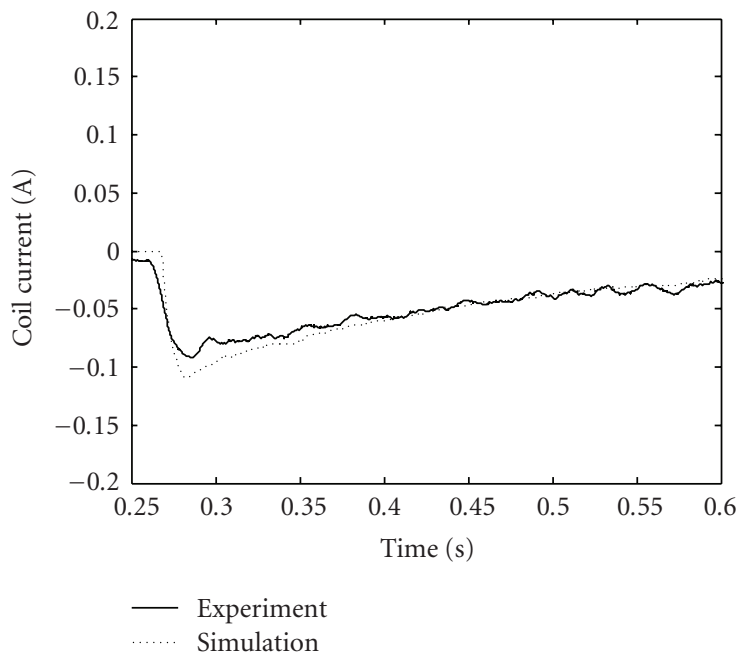

(f)

FIGURE 8: Time-varying waveforms of both armature displacement and coil current during the (a) closing process, (b) holding process, and (c) opening process. 


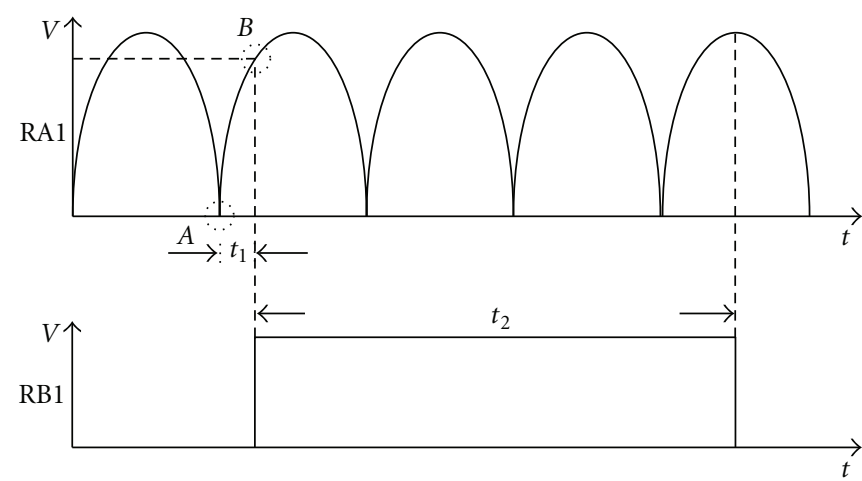

(a)

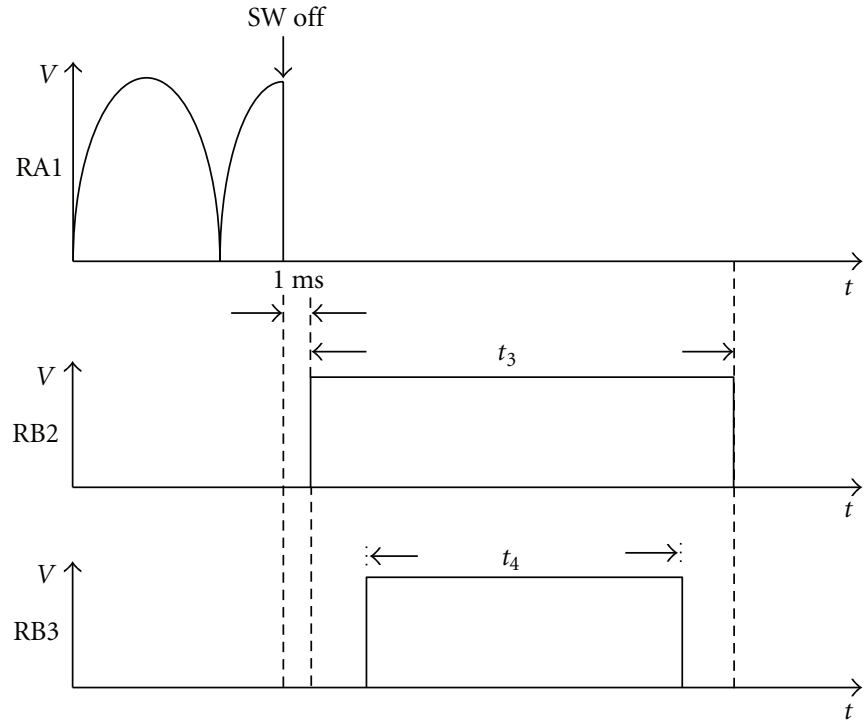

(b)

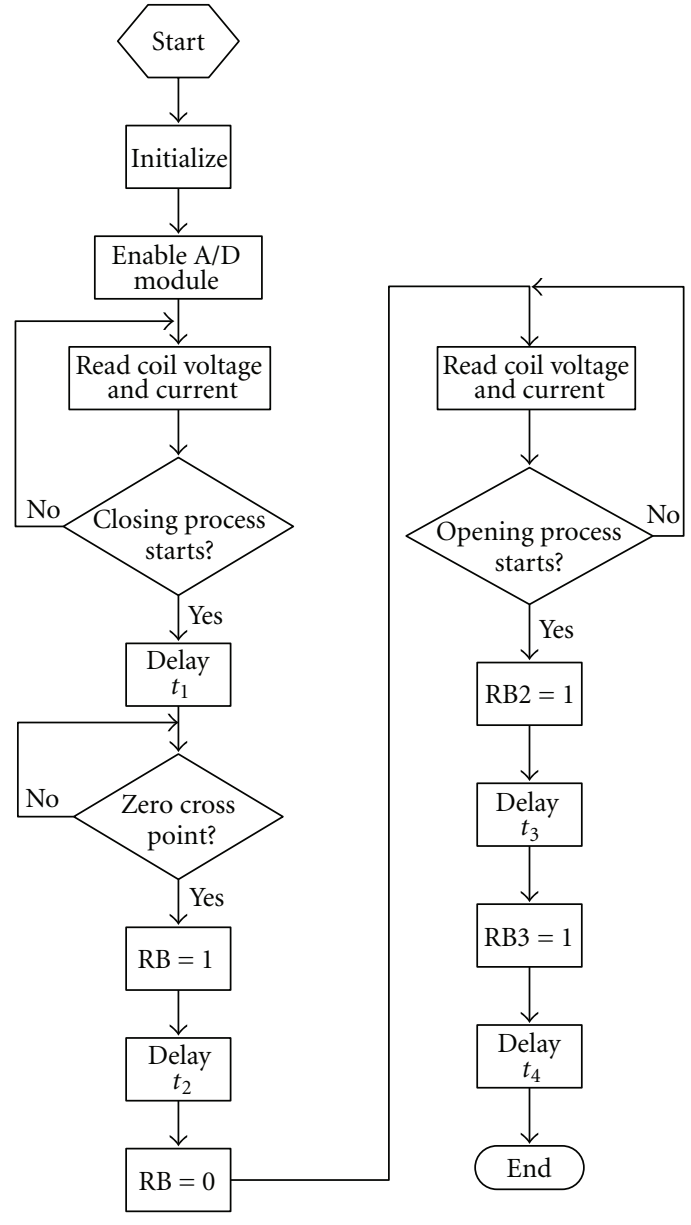

(c)

Figure 9: For AC PM contactor (a) command sequences during the closing process (b) command sequences during the opening process, and (c) flow chart of microcontroller software.

During the opening process, the zero AC voltage source was made sure, and then 1 milliseconds time delay was set for advancing to check whether the opening process of AC PM contactor was actually produced. The pin RB2 of microcontroller became logical high level and delayed for $t_{3}$ time if yes. Similarly, the high logical signal in pin RB3 of microcontroller was generated for assisting the ECU to complete the opening process of AC PM contactor safely.

During the closing process, Figure 10 showed that those corresponding time-varying forces imposed upon armature under different closing phase angles of AC voltage source. The curve showed that when the closing phase angle of applied AC voltage source was set to 30 degree, the resultant magnetic force would be larger than those produced by other closing phase angles. The time-varying velocity of armature of AC PM contactor shown in Figure 10(c) depicted that moving velocity or kinetic energy of armature before two contacts impact was near $0.8 \mathrm{~m} / \mathrm{s}$. The total closing time of contact at 30 degree was obviously shorter than the other cases. Moreover, as those displacement curves of armature shown in Figure 10(b), the closing process of AC
PM contactor would be completed within time interval $0.01 \sim 0.012$ seconds. However, there is a common and important feature occurs after the movable contact of the first time touches the fixed contact, the armature would be accelerated by various accelerations. This result represents that the linear momentum of movable contact after two contacts impact would be dissipated by various rates. This was also the reason why the bounce duration of $\mathrm{AC} P M$ contactor was commonly fewer than that of conventional AC EM contactor. As the moving velocity curves of armature were simulated over a period of possible closing phase angle and shown in Figure 10(d), they were often higher than that in AC EM contactor. These results were generated by the resultant electromagnetic force. It was produced by the addition of original electromagnetic force and holding force of permanent magnet. In addition, the moving velocity curves of armature also showed that they were a periodic function of the closing phase angle of AC voltage source. The AC PM contactor revealed that the period was 180 degree. The moving velocity curve produced at closing phase angle equals 55 degree would lead to a minimum kinetic energy $\left(E_{k}=\left(m v^{2}\right) / 2\right)$ for AC PM contactor. In contrast, 


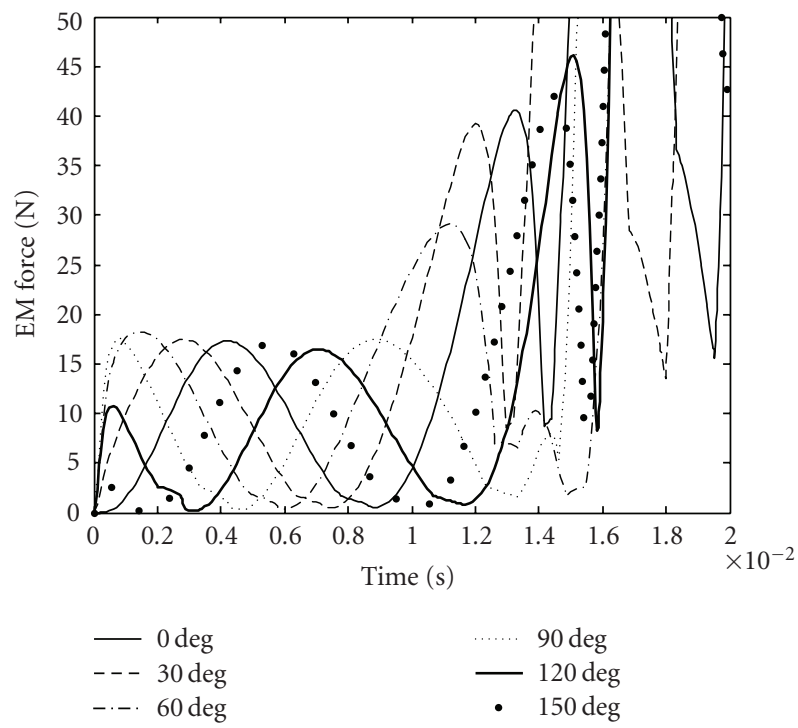

(a)

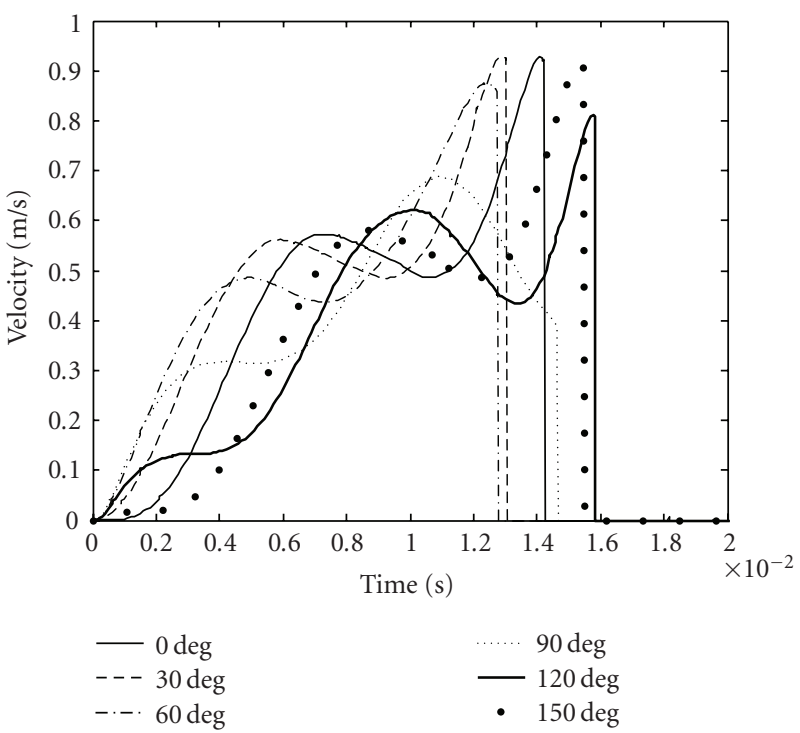

(c)

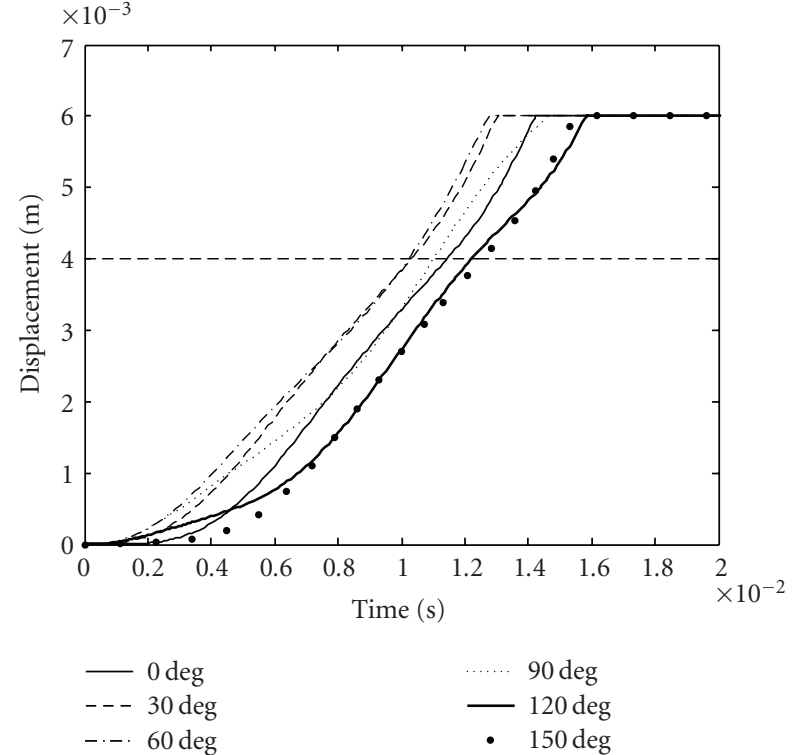

(b)

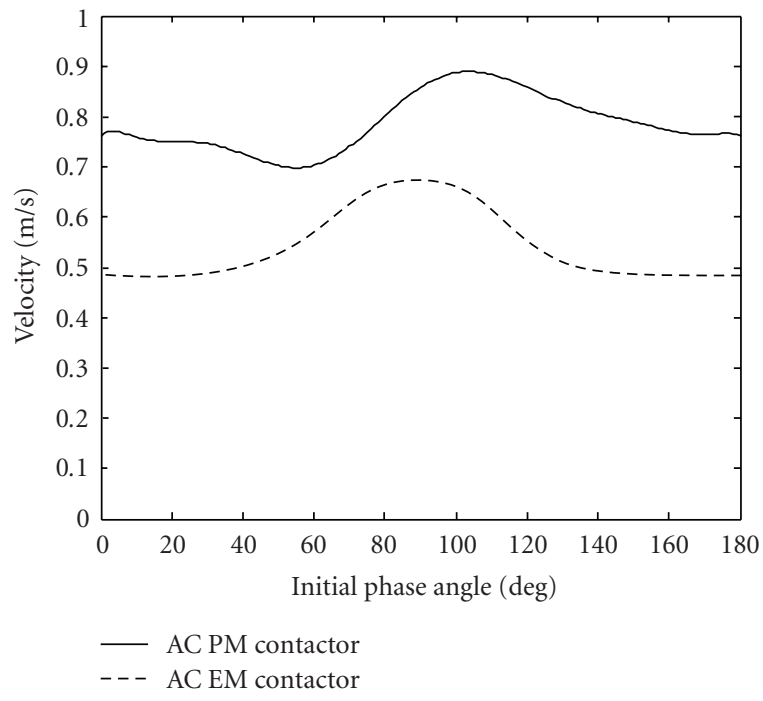

(d)

Figure 10: Time-varying curves of (a) magnetic force, (b) armature displacement, (c) armature velocity, and (d) the angle-varying curve of moving velocity.

an optimal closing phase angle of conventional AC EM contactor also existed at near 0 degree. Of course, this special closing phase angle of applied AC voltage source would also result in minimizing the kinetic energy of armature of AC EM contactor during the closing process as well.

5.3. Assessment of Bouncing Reduction. The designed testing rig had been established and is shown in Figure 11(a). The effects of the proposed bounce reduction technique upon the AC PM contactor were carried out on the testing rig by a series of experiments. One of the three pairs of contact was connected with a circuit including a dc voltage source $E$ and a fixed resistor $R_{t}$, if the voltage $\mathrm{V}_{t}$ across the measuring resistor was set to $E$ the contacts close together; otherwise, the voltage $V_{t}$ across the measuring resistor would be set to zero voltage. There were three types of voltage source: $85 \%, 100 \%$, and $110 \%$ of rated voltage source. They acted as the typical testing voltages of the AC PM contactor in the testing rig, respectively. Aiming at AC PM contactor and AC EM contactor, ten times of closing sequence were conducted and recorded under each of the three testing voltage. For each testing condition, the arithmetic mean of the bouncing times of contact was calculated and is shown in Table 1. Some 


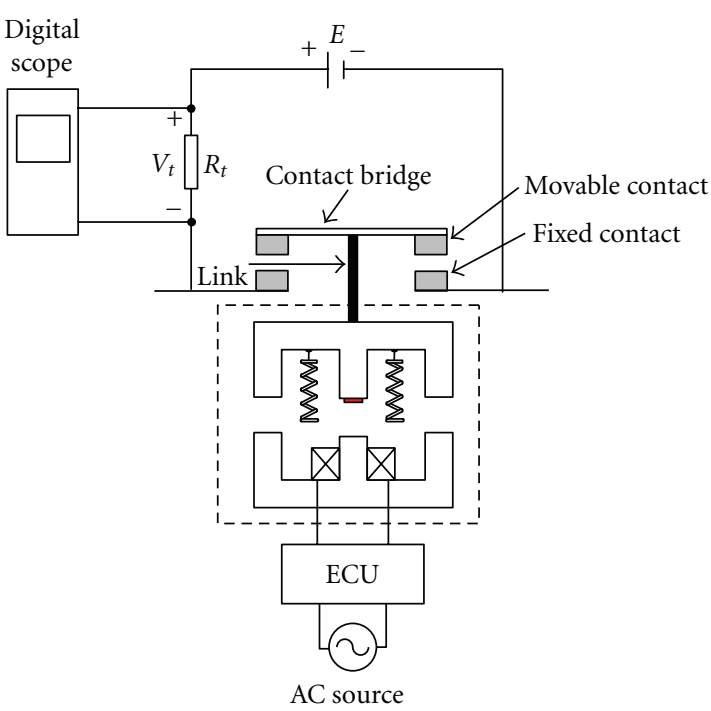

(a)

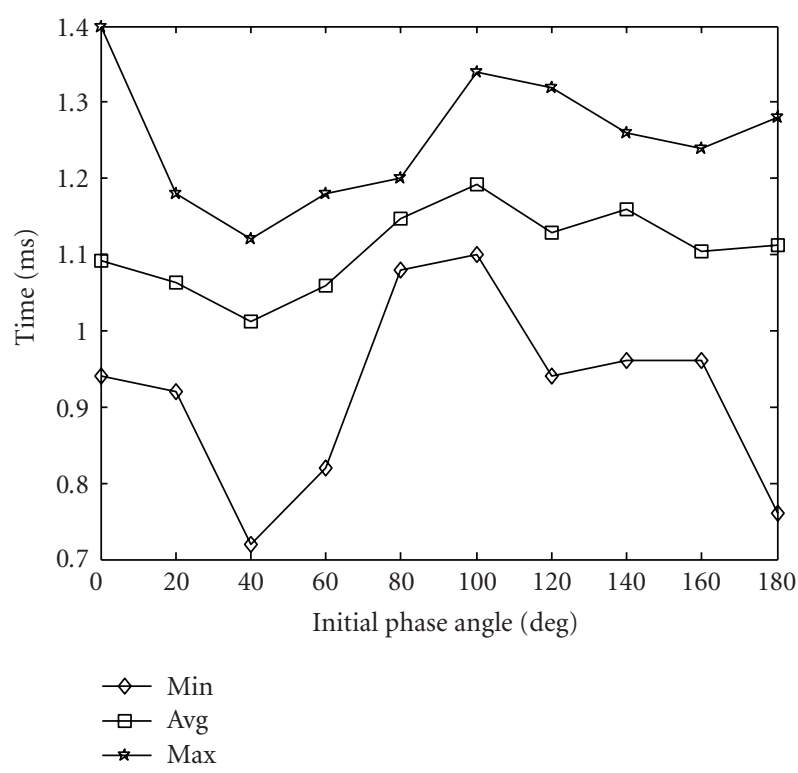

(b)

FIGURE 11: It shows (a) a testing rig for measuring the bouncing duration of contact after two contacts impact (b) measured results.

important conclusions are made based on the above testing results.

(1) Comparing bouncing duration of contact of AC PM contactor with that of AC EM contactor, we can see that AC PM contactor is better.

(2) For any type of contactor, the bouncing duration when a higher value of voltage source was applied would be longer than that of when a lower voltage value of voltage source was applied.

(3) The difference of bounce reduction percentage between AC EM contactor and AC PM contactor was near $38 \%$.

(4) If both AC PM contactor and AC EM contactor were assumed to apply same value of AC voltage source, generally, the bounce-reduction performance obtained by former case was often superior to latter case.

(5) Angle-varying maximum, average, and minimum bounce-duration curves obtained by each testing value of the $\mathrm{AC}$ voltage source were shown in Figure 11(b). The average bounce-duration curve clearly indicated that a minimum moving velocity of armature would be occurred at near 40 degree. Therefore, these experimental results shown in Figure 11(b) are in agreement well with those simulation results shown in Figure 10(d).

5.4. Comparison of Energy Saving. Energy-saving characteristic offered by the AC PM contactor is one of its important advantages. As listed in Table 2, both AC PM contactor and AC EM contactor are assumed to be operated in the holding process for one year. The total energy is dissipated by AC
TABLE 1: Comparisons of average bouncing duration of a pair of contacts after collision for both AC PM contactor and AC EM contactor.

\begin{tabular}{lcc}
\hline Applied AC voltage source & \multicolumn{2}{c}{ Average bouncing duration (ms) } \\
(Nominal value equals 220 V) & EM contactor & PM contactor \\
\hline $85 \%$ & 1.556 & 0.832 \\
$100 \%$ & 1.568 & 1.012 \\
$110 \%$ & 1.620 & 1.088 \\
\hline Percentage of bounce reduction & \multicolumn{3}{c}{$38.19 \%$} \\
\hline
\end{tabular}

TABLe 2: Compare the energy-saving performance of AC EM contactor with AC PM contactor.

\begin{tabular}{lcc}
\hline Item & EM type & PM type \\
\hline Applied voltage (V) & 220 & 220 \\
Coil current (A) & 0.0791 & 0.0138 \\
Volt-Ampere (VA) & 17.402 & 3.036 \\
Total energy (KWH) & 152.44152 & 26.59536 \\
Fee (NT dollars) & 457.32456 & 79.78608 \\
\hline
\end{tabular}

PM contactor is only $27 \%$ of that by AC EM contactor. Moreover, the number of coils equals 2000 turns, which is approximately half of the currently needed coil windings of AC EM contactor.

\section{Conclusions}

An AC PM contactor with ECU controlled actuator is presented and features energy saving and no noise pollution, and voltage-sag immunity. By arranging a permanent magnet on armature, all operations of the AC PM contactor are automatically controlled by the ECU. There is no any 
operating change for those people who are used to the conventional AC EM contactor. But several key problems generally produced by conventional AC EM contactor are then overcome. Based on selecting an optimal closing phase angle of the applied AC voltage source, movable contact of contactor engages with its fixed contact by a minimum kinetic energy. The bouncing duration and energy-saving performance of the proposed AC PM contactor have been validated through some simulation and experimental tests. The bounce duration after two contacts collision during the closing process is then reduced significantly. The energy dissipation is saved very obviously. In addition, the lifespan of contactor contacts is prolonged and their operating reliability is improved as well.

\section{Acknowledgment}

This work was supported by the National Science Council (Taiwan) under Grant NSC 97-2622-E-270-002-CC3.

\section{References}

[1] X. A. Morera and A. G. Espinosa, "Modeling of contact bounce of ac contactor," in Proceedings of the 5th International Conference on Electrical Machines and Systems, vol. 1, pp. 174177, Shengyang, China, August 2001.

[2] T. S. Davies, H. Nouri, and F. W. Britton, "Towards the control of contact bounce," IEEE Transactions on Components Packaging and Manufacturing Technology Part A, vol. 19, no. 3, pp. 353-359, 1996.

[3] J. H. Kiely, H. Nouri, F. Kalvelage, and T. S. Davies, "Development of an application specific integrated circuit for reduction of contact bounce in three phase contactors," in Proceedings of the 46th IEEE Holm Conference on Electrical Contacts, pp. 120129, September 2000.

[4] M. Rong, J. Lou, Y. Liu, and J. Li, "Static and dynamic analysis for contactor with a new type of permanent magnet actuator," IEICE Transactions on Electronics, vol. E89-C, no. 8, pp. 12101216, 2006.

[5] S. Fang and H. Lin, "Magnetic field analysis and control circuit design of permanent magnet actuator for AC contactor," in Proceedings of the 8th International Conference on Electrical Machines and Systems (ICEMS '05), vol. 1, pp. 280-283, 2005.

[6] S. H. Fang, H. Y. Lin, C. F. Yang, X. P. Liu, and J. A. Guo, "Comparison evaluation for permanent magnet arrangements of AC permanent magnet contactor," in Proceeding of International Conference on Electrical Machines and Systems (ICEMS '07), pp. 939-942, Seoul, Korea, October 2007.

[7] D. Holliday and R. Resnick, Fundamental of Physics, John Wiley \& Sons, Taiwan.

[8] W. Li, J. Lu, H. Guo, W. Li, and X. Su, "AC contactor making speed measuring and theoretical analysis," in Proceedings of the 50th IEEE Holm Conference on Electrical Contacts and the 22nd International Conference on Electrical Contacts, pp. 403-407, 2004.

[9] X. Zhou, L. Zou, and E. Hetzmannseder, "Asynchronous modular contactor for intelligent motor control applications," in Proceedings of the 51st IEEE Holm Conference on Electrical Contacts, pp. 55-61, September 2005.

[10] X. Su, J. Lu, B. Gao, G. Liu, and W. Li, "Determination of the best closing phase angle for AC contactor based on game theory," in Proceedings of the 52nd IEEE Holm Conference on Electrical Contacts, pp. 188-192, Montreal, Canada, July 2006.

[11] Z. Xu and P. Zhang, "Intelligent control technology of AC contactor," in Proceedings of the IEEE Power Engineering Society Transmission and Distribution Conference, pp. 1-5, April 2005.

[12] A. E. Fitzgerald, C. Kingsley Jr., and S. D. Umans, Electric Machine, McGraw-Hill, Taiwan, 1983. 

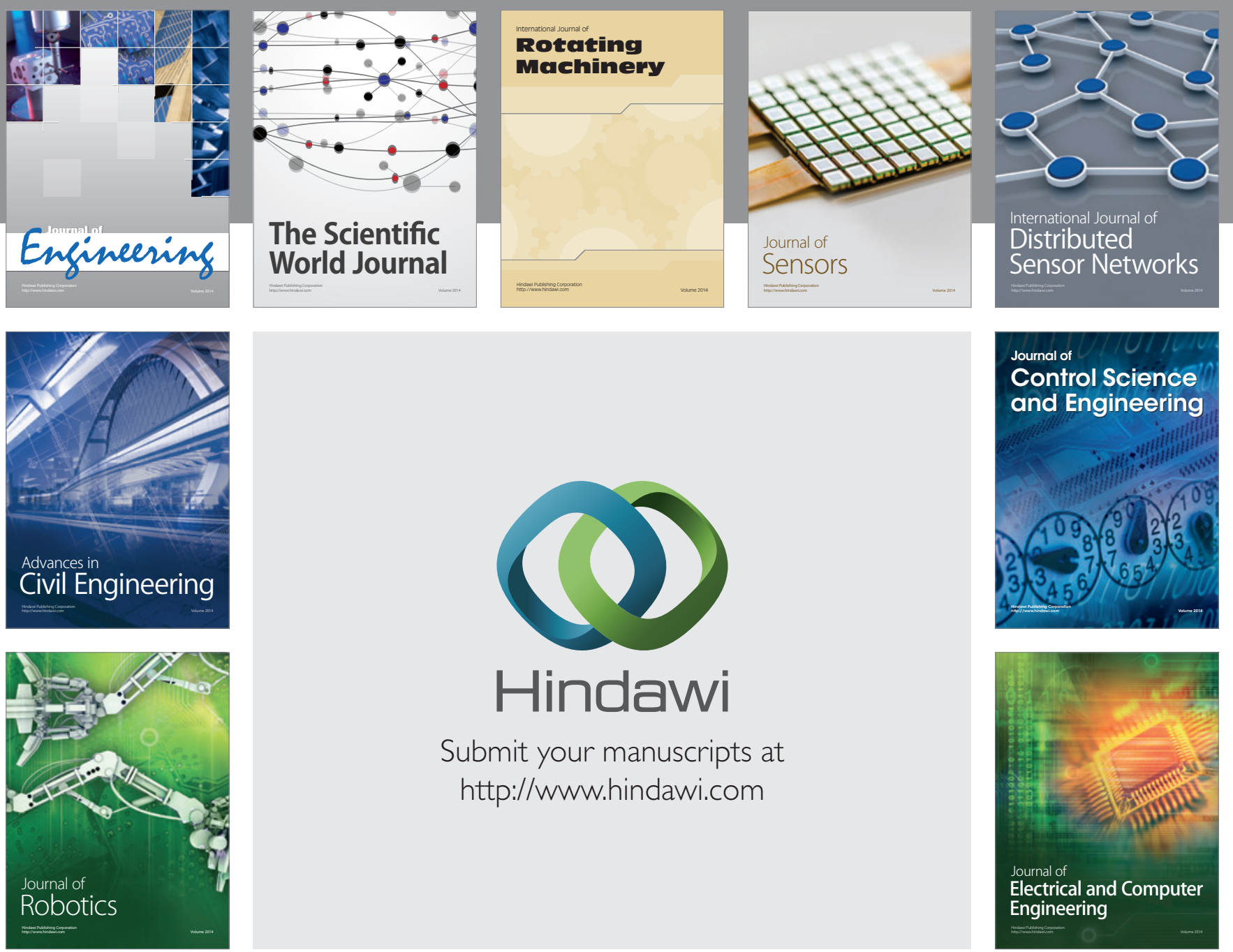

Submit your manuscripts at

http://www.hindawi.com
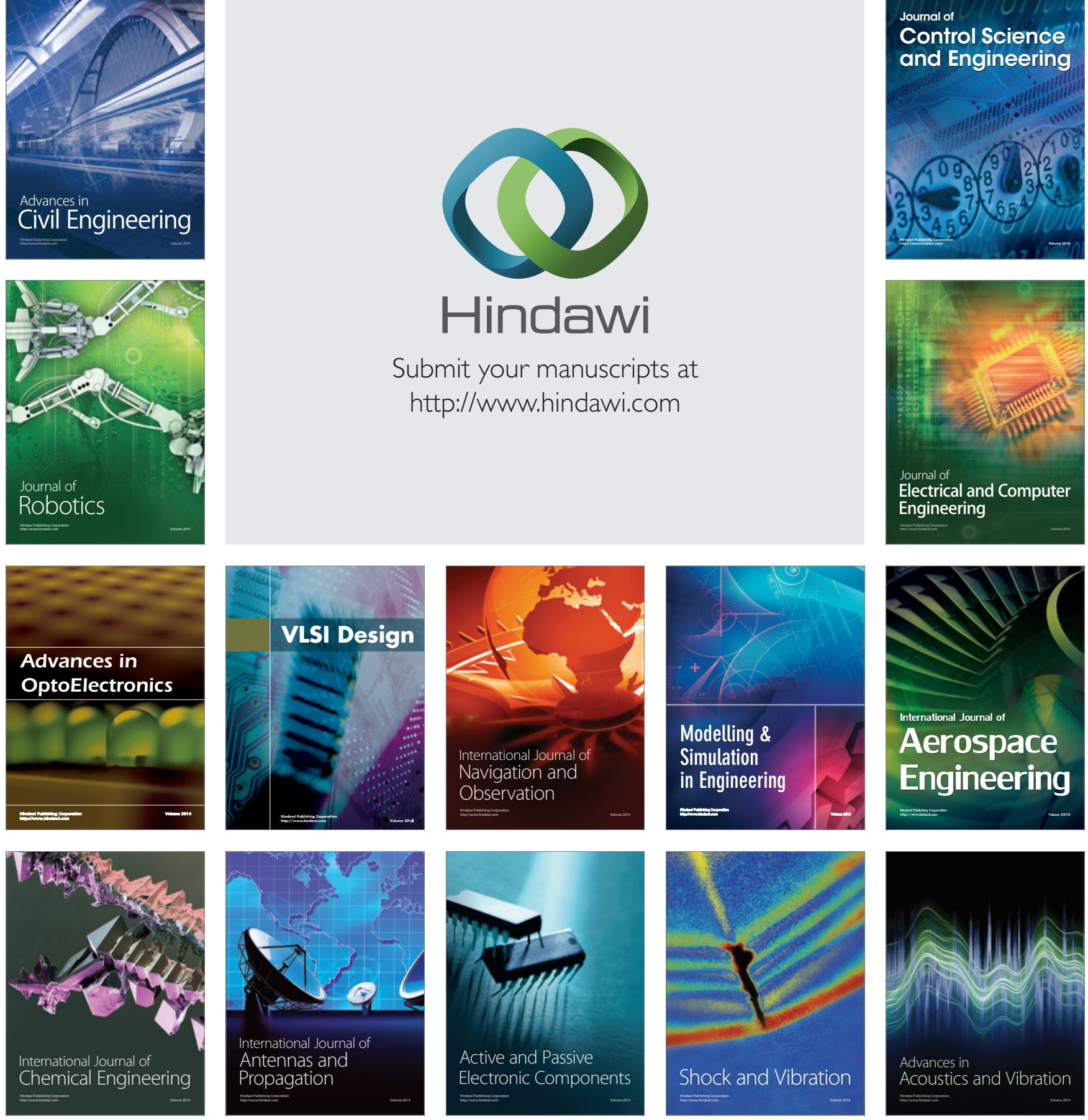